\title{
On snarks that are far from being 3-edge colorable
}

\author{
Jonas Hägglund \\ Department of Mathematics and Mathematical Statistics \\ Umeå University \\ SE-901 87 Umeå, Sweden \\ jonas.hagglund@math.umu.se
}

Submitted: Jun 4, 2013; Accepted: Mar 26, 2016; Published: Apr 15, 2016

Mathematics Subject Classifications: 05C38, 05C70

\begin{abstract}
In this note we construct two infinite snark families which have high oddness and low circumference compared to the number of vertices. Using this construction, we also give a counterexample to a suggested strengthening of Fulkerson's conjecture by showing that the Petersen graph is not the only cyclically 4-edge connected cubic graph which require at least five perfect matchings to cover its edges. Furthermore the counterexample presented has the interesting property that no 2-factor can be part of a cycle double cover.
\end{abstract}

\section{Introduction}

A cubic graph is said to be colorable if it has a 3-edge coloring and uncolorable otherwise. A snark is an uncolorable cubic cyclically 4-edge connected graph. It it well known that an edge minimal counterexample (if such exists) to some classical conjectures in graph theory, such as the cycle double cover conjecture [15, 18], Tutte's 5-flow conjecture [19] and Fulkerson's conjecture [4], must reside in this family of graphs.

There are various ways of measuring how far a snark is from being colorable. One such measure which was introduced by Huck and Kochol [8] is the oddness. The oddness of a bridgeless cubic graph $G$ is defined as the minimum number of odd components in any 2 -factor in $G$ and is denoted by $o(G)$. Another measure is the resistance of $G$, $r_{3}(G)$, which was introduced by Steffen [17] and is defined as the minimal number of edges that needs to be removed from $G$ in order to obtain a 3-edge colorable graph, i.e. $r_{3}(G):=\min \left\{|M|: M \subset E(G)\right.$ and $\left.\chi^{\prime}(G-M)=3\right\}$. It is easy to see that $r_{3}(G) \leqslant o(G)$. It is also known that there exists families of snarks where these measures are arbitrary large and arbitrary far apart [17].

Although snarks can be arbitrary far from being colorable in the sense of oddness and resistance, this might not be the case when we consider other uncolorablilty measures. 
The perfect matching index $\tau(G)$ of a cubic graph $G$ was introduced in [3] and is defined as the minimum number of perfect matchings needed to cover $E(G)$. A famous conjecture by Fulkerson asserts that every bridgeless cubic graph has a double cover by six perfect matchings [4], and if true, would imply that $\tau(G) \leqslant 5$ for every bridgeless cubic graph $G$. Recently Mazzuoccolo showed that these two statements are in fact equivalent [12]. However it is not known if there exists a constant $k$ such that $\tau(G)<k$ for all snarks $G$.

It is easy to see that $\tau(P)=5$, where $P$ is the Petersen graph. It has been proposed $[13,3]$ that a possible strengthening of Fulkerson's conjecture could be the assertion that the Petersen graph is in fact the only snark with this property and that all other snarks have $\tau=4$.

Conjecture 1.1 ( $[13,3])$. Let $G$ be a cyclically 4-edge connected cubic graph. If $\tau(G)=5$, then $G$ is the Petersen graph.

In this paper we present a counterexample to this conjecture. Furthermore we note that this counterexample has the interesting property that no 2-factor can be part of a cycle double cover. We also give simple constructions for two infinite families of snarks with high oddness and resistance compared to the number of vertices.

\section{The construction}

The following simple lemma is well known (see e.g. $[9,14,17])$ and very useful when studying edge colorability of cubic graphs.

Lemma 2.1 (Parity lemma). Let $\phi: E(G) \rightarrow\{1,2,3\}$ be a 3-edge-coloring of a cubic graph $G$. Then for every edge cut $M$ in $G$ we have that $\left|\phi^{-1}(1) \cap M\right| \equiv\left|\phi^{-1}(2) \cap M\right| \equiv$ $\left|\phi^{-1}(3) \cap M\right| \equiv|M|(\bmod 2)$

Following the notation from [10] we say that a semiedge is an edge which is incident to exactly one vertex or one vertex and another semiedge. In the latter case we simply identify it with a normal edge. A multipole $M$ is a triple $M=(V, E, S)$ where $V=V(M)$ is the vertex set, $E=E(M)$ is the edge set and $S=S(M)$ is the set of semiedges. A multipole with $k$ semiedges is called a $k$-pole.

Let $B$ be the 4-pole obtained by removing two adjacent vertices from the Petersen graph (see Figure 1).

Lemma 2.2. In every 3-edge coloring of the 4-pole $B$ the semiedges $a_{1}$ and $a_{2}$ receive the same color.

Proof. Assume that $a_{1}$ and $a_{2}$ have different colors in a 3-edge coloring $\phi$ of $B$. W.l.o.g. we may assume that $\phi\left(a_{1}\right)=1$ and $\phi\left(a_{2}\right)=2$. Now, by Lemma 2.1, one of $b_{1}$ and $b_{2}$ must have color 1 and the other color 2. W.l.o.g. we can assume that $\phi\left(b_{1}\right)=1$ and $\phi\left(b_{2}\right)=2$. Furthermore we can assume w.l.o.g. that $\phi\left(v_{1} v_{2}\right)=2$ and $\phi\left(v_{2} v_{3}\right)=3$. Then $\phi\left(v_{2} v_{6}\right)=3$, but since one of the edges $v_{6} v_{7}$ and $v_{5} v_{6}$ must have color 3 , we have a contradiction. 


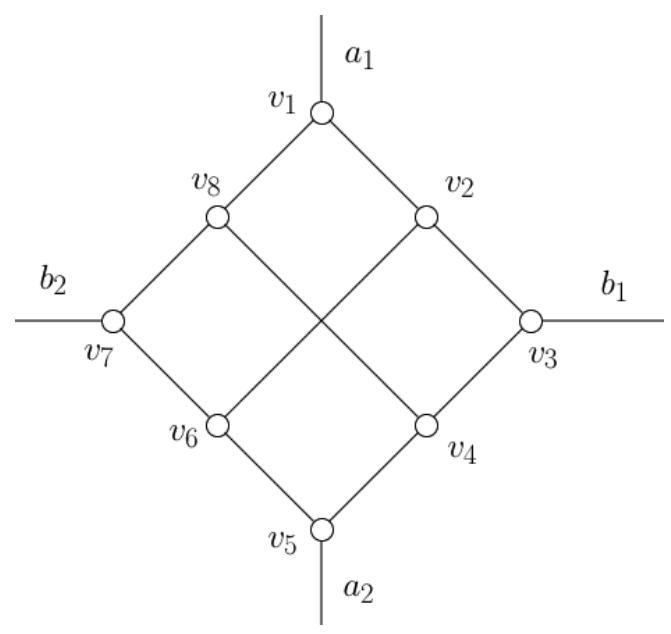

Figure 1: The 4-pole $B$ is constructed from the Petersen graph by removing two adjacent vertices.

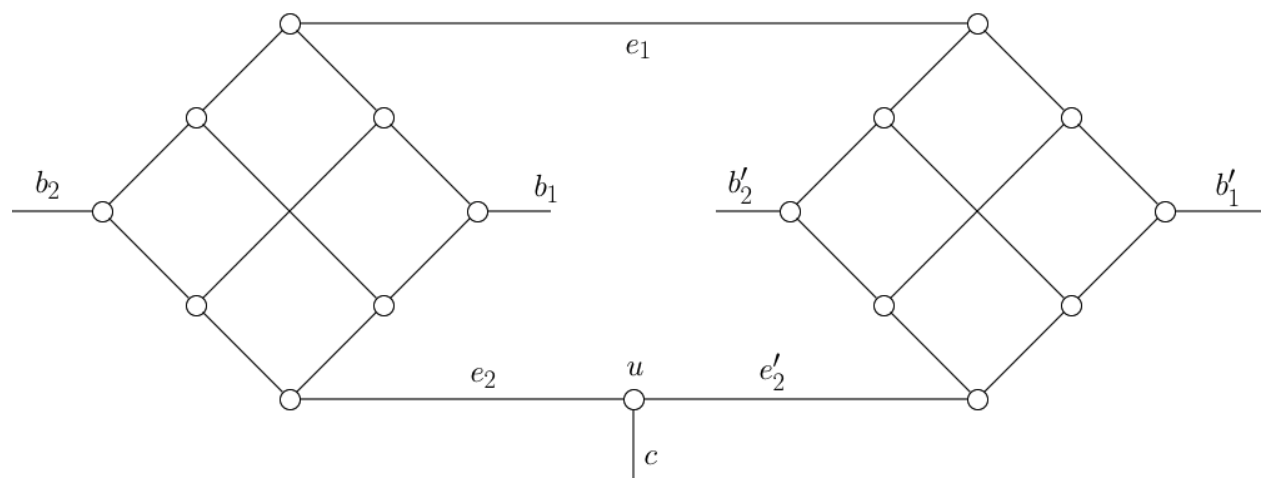

Figure 2: The 5-pole $H_{1}$.

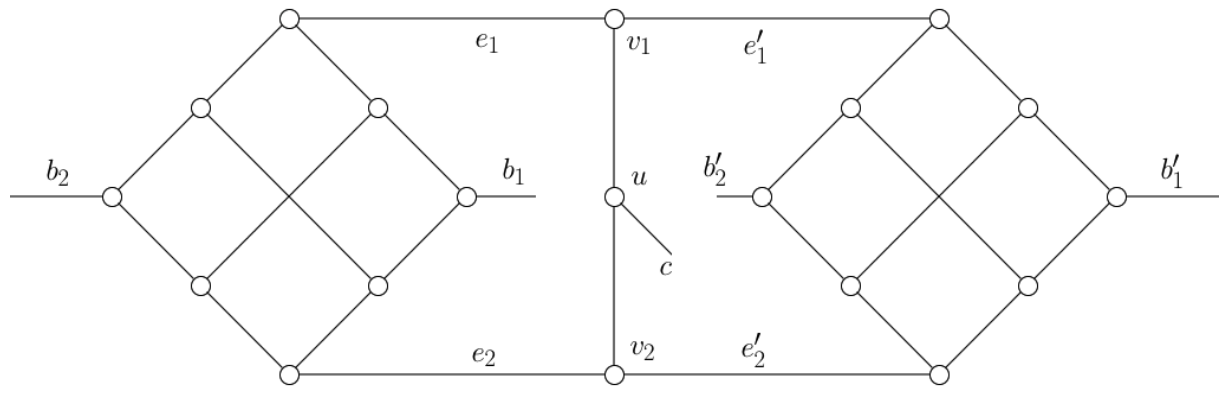

Figure 3: The 5-pole $H_{2}$.

Now consider the 5 -pole $H_{1}$ obtained the following way: Take two copies $B_{1}$ and $B_{2}$ of $B$ and identify the semiedges $b_{1}$ from $B_{1}$ with $b_{1}$ from $B_{2}$ to get an edge $e_{1}$. Then add 
a vertex $u$ which is connected with the semiedges $b_{2}$ from both $B_{1}$ and $B_{2}$ and denote the corresponding new edges by $e_{2}$ and $e_{2}^{\prime}$ (see Figure 2). It is straightforward to verify that this 5-pole cannot be 3-edge colorable.

Lemma 2.3. $H_{1}$ is not 3-edge colorable.

Proof. Assume the opposite. Then by Lemma $2.2 e_{1}$ must have the same color as $e_{2}$, but $e_{1}$ must also have the same color as $e_{2}^{\prime}$ which is impossible since $e_{2}$ and $e_{2}^{\prime}$ are incident edges.

The 5-pole $H_{2}$ is formed in a similar way by joining two copies of $B$ with three vertices $v_{1}, v_{2}$ and $u$ as shown in Figure 3.

Lemma 2.4. $\mathrm{H}_{2}$ is not 3-edge colorable.

Proof. Assume the the graph has a 3-edge coloring. Then by Lemma $2.2 e_{1}$ must have the same color as $e_{2}$ and $e_{1}^{\prime}$ must have the same color as $e_{2}^{\prime}$. W.l.o.g. we may assume that $e_{1}$ and $e_{2}$ has color 1 and $e_{1}^{\prime}$ and $e_{2}^{\prime}$ has color 2. But then both $v_{1} u$ and $v_{2} u$ must have color 3, which is impossible.

We can now use that fact that any cubic graph which contains either $H_{1}$ or $H_{2}$ as a subgraph cannot be colorable to create two infinite families of snarks.

\section{Construction 1.}

Let $G$ be any 2-edge connected cubic graph and let $D$ be a 2-regular subgraph of $G$. Let $C=\left(v_{1}, v_{2}, \ldots, v_{k}\right)$ be a cycle in $\mathrm{D}$. Now, form a graph by removing all edges $v_{i} v_{i+1}$ (indices are taken modulo $k$ ). Then add $k$ copies $B_{1}, \ldots, B_{k}$ of the 4 -pole $B$ and denote the semiedge edges in $B_{i}$ by $a_{l}^{i}, b_{l}^{i}$ for $l \in\{1,2\}$. Now, for all $i \in\{1, \ldots, k\}$, connect $v_{i}$ with the semiedge $b_{1}^{i}$, the semiedges $a_{2}^{i}$ with $b_{2}^{i+1}$ and $a_{1}^{i}$ with $v_{i+1}$. Repeat this process for every cycle in $D$ and denote the resulting proper graph by $G^{\prime}$.

We call $G^{\prime}$ a semi blowup of $(G, D)$ and denote $G^{\prime}$ by $\operatorname{SemiBlowup}(G, D)$.

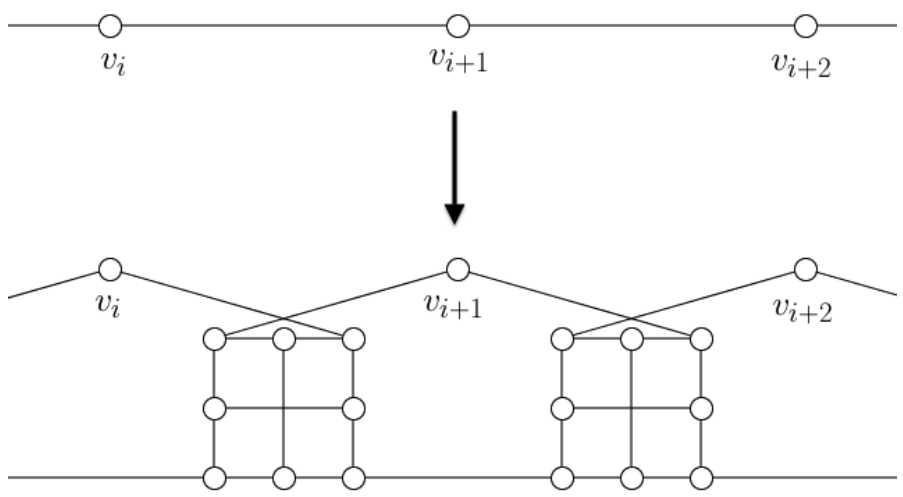

Figure 4: An illustration of Construction 1. 


\section{Construction 2.}

The second construction is similar to the previous one. Let $G$ be any 2-edge connected cubic graph and let $D$ be a 2-regular subgraph of $G$. Furthermore let $C=\left(v_{1}, v_{2}, \ldots, v_{k}\right)$ be a cycle in $D$ and remove all edges $v_{i} v_{i+1}, i=1, \ldots, k$ and add $k$ copies $B_{1}, \ldots, B_{k}$ of $B$. Now add two additional vertices $u_{i}$ and $w_{i}$. Then add the edges $\left\{v_{i} u_{i}, v_{i} w_{i}\right\}$ and form edges from the semiedges by connecting $u_{i}$ with $b_{1}^{i}, w_{i}$ with $b_{2}^{i}, a_{1}^{i}$ with $u_{i+1}$ and $a_{2}^{i}$ with $w_{i+1}$. We continue this process for every cycle in $D$ and call the resulting graph $G^{\prime \prime}$. We say that $G^{\prime \prime}$ a blowup of $D$ and $G^{\prime \prime}$ is denoted by $\operatorname{Blowup}(G, D)$.

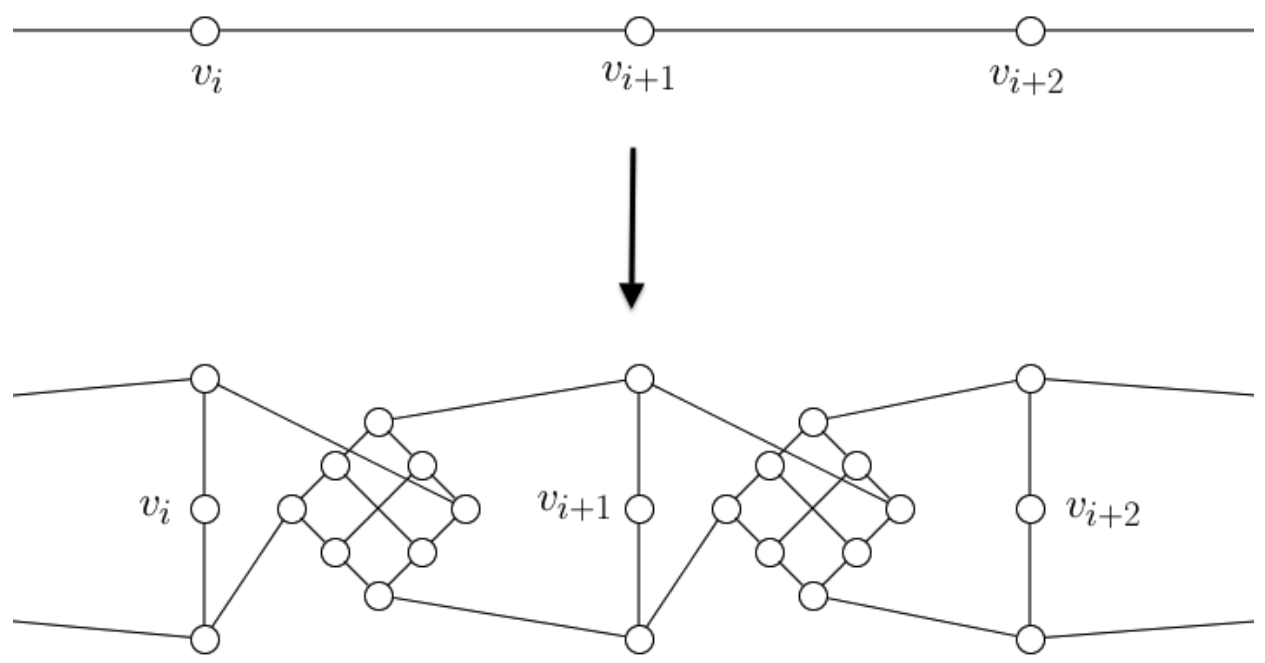

Figure 5: An illustration of construction 2.

Theorem 2.5. Let $G$ be a 3-edge connected cubic graph with a 2-regular subgraph $D$. Then Blowup $(G, D)$ and SemiBlowup $(G, D)$ are not 3-edge colorable. Furthermore if $D_{1}, \ldots, D_{l}$ are the disjoint cycles of $D$, we have that

$$
o(\operatorname{Blowup}(G, D)) \geqslant r_{3}(\operatorname{Blowup}(G, D)) \geqslant \sum_{i=1}^{l}\left\lceil\frac{\left|V\left(D_{i}\right)\right|}{2}\right\rceil
$$

and the same inequality holds for $\operatorname{SemiBlowup}(G, D)$.

Proof. Let $G_{1}:=\operatorname{SemiBlowup}(G, D)$ and $G_{2}:=\operatorname{Blowup}(G, D)$. Every pair of adjacent edges on a cycle in $D$ gives a subgraph isomorphic to $H_{1}$ in $G_{1}$ and a subgraph isomorphic to $H_{2}$ in $\operatorname{SemiBlowup}(G, D)$. Since a cubic graph cannot be colorable if either $H_{1}$ or $H_{2}$ are subgraphs, we have that $r_{3}\left(G_{1}\right)$ and $r_{3}\left(G_{2}\right)$ is at least the number of edges that needs to be removed in order to make $G_{1} H_{1}$-free or $G_{2} H_{2}$-free. It is easy to see that this is at least $\sum_{i=1}^{l} \beta\left(D_{i}\right)$ where $\beta$ is the vertex cover number of a graph (the minimum number of vertices needed to cover all the edges in a graph). Since an optimal vertex cover of an 
even cycle is to choose every second vertex we have that $\beta\left(C_{2 k}\right)=k$ and for odd cycles at least two adjacent vertices must be in the vertex cover, so $\beta\left(C_{2 k+1}\right)=k+1$. Hence

$$
o\left(G_{j}\right) \geqslant r_{3}\left(G_{j}\right) \geqslant \sum_{i=1}^{l} \beta\left(D_{i}\right)=\sum_{i=1}^{l}\left\lceil\frac{\left|V\left(D_{i}\right)\right|}{2}\right\rceil
$$

for $j=1,2$.

If $G$ has no cyclic $k$-edge-cuts where $k \leqslant 3$, then both blowup and semiblowup will produce snarks. This also gives a simple construction of cyclically 4-edge connected snarks with rather high oddness compared to the number of vertices.

By considering the semiblowup of a hamiltonian cycle in a hamiltonian cubic graph, we get the following:

Corollary 2.6. For every $k \in \mathbb{N}, k>2$ there exists a snark $G_{k}$ with $\left|V\left(G_{k}\right)\right|=18 k$ and $o\left(G_{k}\right) \geqslant k$.

This can be improved slightly by instead use many short cycles of odd lengths.

Corollary 2.7. For every $k \in \mathbb{N}$ there exists a snark $G_{k}$ with $\left|V\left(G_{k}\right)\right|=90 k$ and $o\left(G_{k}\right) \geqslant$ $6 k$.

Proof. Given $k \in \mathbb{N}$, let $F_{k}$ be the 2-factor formed from $2 k$ copies of $C_{5}$. Then add $10 k$ edges between the cycles in $F$ in order to obtain a cubic graph $G_{k}^{\prime}$. Now let $G_{k}:=$ SemiBlowup $\left(G^{\prime}, F\right)$. Since $\beta\left(C_{5}\right)=3$ we get $o\left(G_{k}\right) \geqslant 2 k \beta\left(C_{5}\right)=6 k$.

In Corollary 2.7 the oddness of $G_{k}$ grows linearly in the number of vertices. Obviously it is impossible to construct a family of snarks where the oddness grows superlinearly. Let

$$
q_{k}:=\max \left\{\frac{k}{|V(G)|}: G \in \mathcal{G} \text { and } o(G) \geqslant k\right\}
$$

where $\mathcal{G}$ is the family of snarks. The Petersen graph is the smallest snark so $q_{2}=\frac{1}{5}$. It is known that the smallest snark with oddness 4 has at least 38 vertices [1] and from Theorem 2.5 we can construct a snark with oddness 4 on 46 vertices (use the semiblowup construction on the cubic graph on 6 vertices which consists of a 5-cycle, a cord and a $K_{1,3}$ ). Hence, $\frac{2}{23} \leqslant q_{4} \leqslant \frac{2}{19}$. From Corollary 2.7 we get $q_{6 k} \geqslant \frac{1}{15}$ and from Corollary 2.6 we get $q_{k} \geqslant \frac{1}{18}$. We pose the following two problems:

Problem 1. Let $k$ be a given even number. Determine $q_{k}$ when $k>2$.

Problem 2. What is the largest value $c$ such that $q_{k} \geqslant c$ for all even $k$. 


\section{$3 \quad$ Perfect matching covers}

Let $C$ be a cycle of length 3 in $K_{4}$ and consider the graph $\operatorname{Blowup}\left(K_{4}, C\right)$. By using a computer, it is easy to see that this graph has perfect matching index 5 . Note that this graph was also observed in [1], as an example of a snark of minimum order for which the removal of any vertex yields a graph homeomorphic to a non 3-edge colorable cubic graph.

Observation 3.1. $\tau\left(\operatorname{Blowup}\left(K_{4}, C\right)\right)=5$.

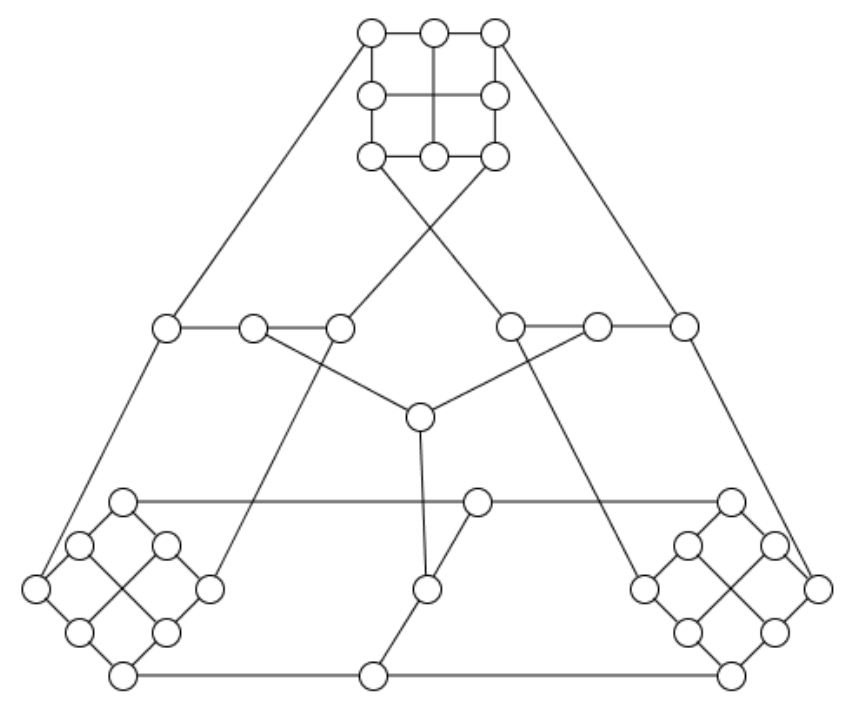

Figure 6: An illustration of $\operatorname{Blowup}\left(K_{4}, C\right)$.

It is possible to construct other snarks with this property. Another example is the blowup of a 4-cycle in the prism depicted in Figure 7. However we do not have any good characterization of the cubic graphs and 2-regular subgraphs for which the blowupconstruction yields snarks with perfect matching index 5 .

Problem 3. Is it possible to give a simple characterization of cubic graphs $G$ with $\tau(G)=$ $5 ?$

Graphs constructed with the blowup-construction always contains cyclic 4-edge cuts, and other than the Petersen graph, we do not know of any cyclically 5-edge connected snarks $G$ with $\tau(G)=5$.

Problem 4 . Are there any cyclically 5 -edge connected snarks $G$, different from the Petersen graph, such that $\tau(G)=5$ ?

\section{Cycle double covers}

We say that a cycle double cover (abbreviated CDC) of a graph is multiset of cycles such that every edge is covered by exactly two cycles. A $k-C D C$ is a CDC where we can color 


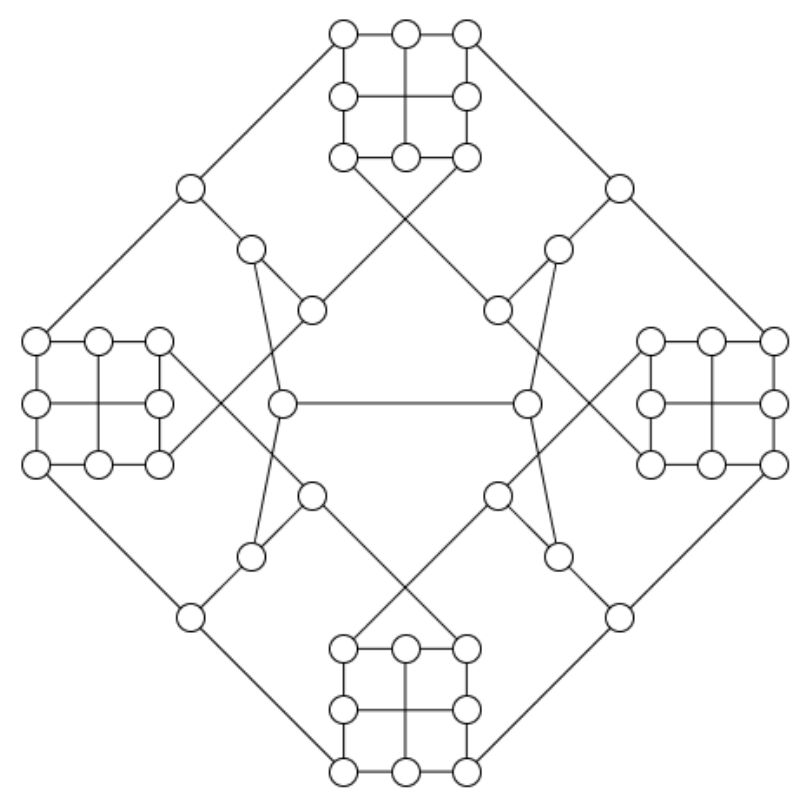

Figure 7: An illustration of $\operatorname{Blowup}\left(\right.$ Prism, $\left.C_{4}\right)$.

assign $k$ colors to the cycles in such a way that if two cycles share an edge, they will always receive different colors. A famous conjecture by Seymour [15] and Szekeres [18] asserts that every bridgeless cubic graph has a CDC, and a conjecture by Celmis [2] strengthen this further by asserting that every such graph in fact has a 5-CDC. Celmins also observed a connection between the perfect matching index and the existence of a 5-CDC where one color class induces a 2-factor.

Theorem 4.1 (Celmins [2]). Let $G$ be a cubic graph. Then $\tau(G) \leqslant 4$ if and only if $G$ has a 5-CDC where one color class induces a 2-factor in $G$.

From Theorem 4.1 and Observation 3.1 we see that $\operatorname{Blowup}\left(K_{4}, C\right)$, where $C$ is a 3-cycle in $K_{4}$, also has the following interesting property.

Corollary 4.2. No 2-factor in Blowup $\left(K_{4}, C\right)$ can be part of a 5-CDC.

By a computer search we also make the following stronger observation.

Observation 4.3. No 2-factor in Blowup $\left(K_{4}, C\right)$ can be part of a $C D C$.

\subsection{Circumference}

It is known that cubic graphs with oddness 4 or less have cycle double covers $[5,7]$. It is also known that the cycle double cover conjecture holds for graphs with sufficiently long cycles. Given a graph $G$, let the circumference of $G$ be the length of the longest cycle. We denote this by $\operatorname{circ}(G)$. In $[6]$ it is shown that if a cubic graph $G$ has $\operatorname{circ}(G) \geqslant|V(G)|-9$, then $G$ has a cycle double cover. This result was further improved in [1] to $|V(G)|-10$. 
We have already seen that the snarks constructed in Section 2 can have arbitrary high oddness. We will now show that the same constructions also produces snarks with low circumference compared to the number of vertices.

Given a cubic graph $G$, let $\rho(G)$ denote the minimum number of vertices that needs to be removed from $G$ in order to obtain a 3-edge colorable graph. It was shown in [16] that this number equals the resistance of the graph.

Theorem 4.4 (Steffen [16]). Let $G$ be a cubic graph. Then $\rho(G)=r_{3}(G)$.

This theorem was later generalized in [11] to apply to all graphs with vertices of degee at most 3. A simple consequence of this theorem is a bound on the circumference.

Lemma 4.5. Let $G$ be a cubic graph. Then $\operatorname{circ}(G) \leqslant|V(G)|-r_{3}(G)+1$.

Proof. Assume that $G$ has a cycle $C$ with $|V(C)|>|V(G)|-r_{3}(G)+1$. Now consider the graph $G^{\prime}=G-(V(G)-V(C))$. If $|V(C)|$ is even, then this graph is 3-edge colorable, since we can color the edges of $C$ alternating between colors 1 and 2 , and the cords of $C$ with color 3. However this gives $\rho(G)<r_{3}(G)-1$ contradicting Theorem 4.4. If, on the other hand, $|V(C)|$ is odd, then obviously $G^{\prime}-v$ is 3-edge colorable for any vertex $v$ in $C$. We now have $\rho(G)<r_{3}(G)$ which once again contradicts Theorem 4.4.

From Lemma 4.5 and Theorem 2.5 we can see that the graphs obtained from the blowup and semiblowup constructions cannot have too long cycles.

Corollary 4.6. Given a cubic graph $G$ and a 2-regular subgraph $D$ with disjoint cycles $C_{1}, C_{2}, \ldots, C_{k}$, then

$$
\operatorname{circ}(\operatorname{Blowup}(G, D)) \leqslant|V(G)|-\sum_{i=1}^{k}\left\lceil\frac{\left|V\left(C_{i}\right)\right|}{2}\right\rceil+1
$$

and the same holds for SemiBlowup $(G, D)$.

\section{Concluding remarks}

There are other constructions of snarks that are far from 3-edge colorable that are similar to the blowup and semiblowup constructions using other $k$-poles than $B$ (any $k$-pole which is either non-3-edge colorable or where the colors are forced as above works). See e.g. [10], [17] and [14] for more comprehensive studies of this.

It is somewhat unsatisfactory to require the assistance of a computer to show that the blowup of a 3 -cycle in $K_{4}$ has $\tau=5$. Given a good characterization of this property, it might be possible to construct an infinite family of such snarks. 


\section{References}

[1] Gunnar Brinkmann, Jan Goedgebeur, Jonas Hägglund, and Klas Markström. Generation and properties of snarks. J. Combin. Theory Ser. B, 103(4):468-488, 2013.

[2] Uldis Alfred Celmins. On cubic graphs that do not have an edge 3-colouring. ProQuest LLC, Ann Arbor, MI, 1985. Thesis (Ph.D.)-University of Waterloo (Canada).

[3] Jean-Luc Fouquet and Jean-Marie Vanherpe. On the perfect matching index of bridgeless cubic graphs. arXiv:0904.1296.

[4] D. R. Fulkerson. Blocking and anti-blocking pairs of polyhedra. Math. Programming, 1:168-194, 1971.

[5] Roland Häggkvist and Sean McGuinness. Double covers of cubic graphs with oddness 4. J. Combin. Theory Ser. B, 93(2):251-277, 2005.

[6] Jonas Hägglund and Klas Markström. On stable cycles and cycle double covers of graphs with large circumference. Discrete Mathematics, 2011.

[7] Andreas Huck. On cycle-double covers of graphs of small oddness. Discrete Math., 229(1-3):125-165, 2001. Combinatorics, graph theory, algorithms and applications.

[8] Andreas Huck and Martin Kochol. Five cycle double covers of some cubic graphs. J. Combin. Theory Ser. B, 64(1):119-125, 1995.

[9] Rufus Isaacs. Infinite families of nontrivial trivalent graphs which are not Tait colorable. Amer. Math. Monthly, 82:221-239, 1975.

[10] Martin Kochol. Snarks without small cycles. J. Combin. Theory Ser. B, 67(1):34-47, 1996.

[11] Martin Kochol. Three measures of edge-uncolorability. Discrete Math., 311(1):106$108,2011$.

[12] G. Mazzuoccolo. The equivalence of two conjectures of Berge and Fulkerson. J. Graph Theory, 68(2):125-128, 2011.

[13] Giuseppe Mazzuoccolo. Covering a 3-graph with perfect matchings. arXiv:1111.1871.

[14] Roman Nedela and Martin Škoviera. Decompositions and reductions of snarks. J. Graph Theory, 22(3):253-279, 1996.

[15] P. D. Seymour. Sums of circuits. In Graph theory and related topics (Proc. Conf., Univ. Waterloo, Waterloo, Ont., 1977), pages 341-355. Academic Press, New York, 1979.

[16] Eckhard Steffen. Classification and characterizations of snarks. Discrete Math., 188(1-3):183-203, 1998.

[17] Eckhard Steffen. Measurements of edge-uncolorability. Discrete Math., 280(1-3):191$214,2004$.

[18] G. Szekeres. Polyhedral decompositions of cubic graphs. Bull. Austral. Math. Soc., 8:367-387, 1973.

[19] W. T. Tutte. A contribution to the theory of chromatic polynomials. Canadian J. Math., 6:80-91, 1954. 